\title{
Processo de Desenvolvimento de Software: uma Análise Exploratória com Profissionais que Desenvolvem Jogos Eletrônicos Educacionais
}

\author{
João Coelho Neto ${ }^{1}$, Sheila Reinehr ${ }^{2}$, Andreia Malucelli ${ }^{2}$ \\ ${ }^{1}$ Centro de Ciências Humanas e da Educação - Colegiado de Matemática - \\ Universidade Estadual do Norte do Paraná (UENP) - Cornélio Procópio - PR - Brazil \\ ${ }^{2}$ Programa de Pós-Graduação em Informática (PPGIa) - Polytechnic School - Pontifícia \\ Universidade Católica do Paraná (PUCPR) - Curitiba - PR - Brazil \\ joaocoelho@uenp.edu.br, sheila.reinehrepucpr.br, \\ malu@ppgia.pucpr.br
}

\begin{abstract}
The aim of this study is to identify what the software development processes are being used by professionals who develop electronic educational games, and investigate if there is a specific process that involves the cognitive area, since these games can helps in the educational context and it is developed in the multidisciplinary settings. A qualitative study with semistructured questionnaire was used. Based on this information, it was found that most professionals use classic software development processes and there is little discussion of cognitive actions during the development of an electronic educational game.
\end{abstract}

Resumo. O objetivo desse estudo é identificar quais são os processos de desenvolvimento de software que estão sendo utilizados por profissionais que desenvolvem jogos eletrônicos educacionais, além de investigar se existe algum processo de software que envolva a área cognitiva, já que estes jogos eletrônicos educacionais podem auxiliar no âmbito educacional e são desenvolvidos em contextos multidisciplinares. Foi realizada uma pesquisa qualitativa, com questionário semiestruturado de apoio. Com base nas informações obtidas, identificou-se que a maioria dos profissionais utiliza processos de desenvolvimento de software clássicos e há pouca discussão de ações cognitivas durante o desenvolvimento do jogo eletrônico educacional.

\section{Introdução}

$\mathrm{Na}$ área de desenvolvimento de software, esforços têm sido despendidos na melhoria de processos, para que atividades no desenvolvimento de softwares sejam estruturadas, a fim de prever uma qualidade no produto desenvolvido [Leal et al. 2012].

O desenvolvimento de um software, no caso dessa pesquisa, o jogo eletrônico educacional, pode ser extremamente complexo e há várias maneiras de se executar as diversas atividades que fazem parte do processo de desenvolvimento. A definição de um processo pode auxiliar os profissionais a executarem estas atividades, facilitando, assim, seu entendimento e na melhoria na qualidade do software final [Humphrey 1989]. 
Dessa forma, uma metodologia de engenharia de software especializada para o domínio de jogos eletrônicos, em especial para a área educacional, poderia auxiliar nesse sentido [Petrillo 2008], [Callele, Neufeld e Schneider 2005]. Acredita-se que a utilização de um processo de desenvolvimento específico para uma proposta educacional, que trabalha as ações educacionais e cognitivas em conjunto, possa auxiliar a equipe a identificar e contextualizar essas ações educacionais durante 0 desenvolvimento do produto educacional final.

Utilizar um processo para o desenvolvimento de jogos eletrônicos não é trivial, visto que conforme as empresas adquirem experiência, passam a modelar um processo próprio, que com o tempo, vai sendo aperfeiçoado para atender às necessidades encontradas, e com a competitividade do mercado, esse conhecimento não é compartilhado. O acesso à metodologia de desenvolvimento é então restrito, e os desenvolvedores de jogos costumam, por exemplo, não incluir em seu escopo o planejamento, partindo direto para sua implementação, o que tem como consequência altos níveis de retrabalho [Santos, Góes e Almeida 2005].

Diante deste contexto, este trabalho vem apresentar os resultados de uma pesquisa qualitativa e exploratória, com profissionais de desenvolvimento de jogos eletrônicos diversos e educacionais. $\mathrm{O}$ artigo tem como objetivo identificar de que forma estão sendo desenvolvidos os jogos eletrônicos por estes profissionais, e se há algum processo específico para a área educacional.

Este artigo foi divido em cinco seções, a primeira seção contextualiza a temática; na segunda seção uma contextualização teórica é apresentada; na terceira seção a abordagem metodológica é descrita; a quarta seção apresenta e discute os resultados e a quinta seção finaliza o trabalho.

\section{Desenvolvimento de Jogos Eletrônicos Educacionais}

No contexto do processo de desenvolvimento de software, principalmente, os de jogos eletrônicos, o uso de processos de desenvolvimento para jogos eletrônicos tanto diversos quanto educacionais é raro [Santos, Góes e Almeida 2012].

Esse panorama é apresentado em uma pesquisa feita por Pietruchinski et al. (2011) em um evento específico na área de informática na educação, para verificar os tipos de jogos eletrônicos que estão sendo produzidos. Verificou-se que, o percentual das publicações de artigos contemplando essa temática foi de 5,4\% e, com relação à questão de processos para jogos eletrônicos foi encontrado um artigo resumido, Silva, Calisto e Barbosa (2010) que contemplava um processo para desenvolvimento de jogos eletrônicos educacionais, porém, sem mencionar-se com as questões educacionais (cognitivas) em suas etapas.

A área de desenvolvimento e uso de jogos eletrônicos educacionais estão em crescimento [Ampatzoglou e Stamelos 2010] e há necessidade de uma maior discussão e conhecimento das questões educacionais envolvidas neste processo, visto que essa discussão é de extrema importância.

Desta maneira, se um jogo eletrônico educacional for desenvolvido apoiado em questões educacionais e cognitivas, pode resultar em atividades agradáveis, com objetivos educacionais especializados para desenvolver a aprendizagem [Silva, Calisto, 
Barbosa 2010] e [Pinto et al. 2008], tornando-se assim, um poderoso recurso para a construção do conhecimento e do desenvolvimento de habilidades cognitivas.

Portanto, se essas questões educacionais e cognitivas forem identificadas pelas equipes de desenvolvimento e transpostas nas atividades de desenvolvimento, poderão auxiliar tanto o profissional de jogos quanto a equipe multidisciplinar na compreensão de ações educacionais significativas, e como resultado, atividades educacionais que são formalizadas por meio do jogo eletrônico educacional desenvolvido [Petrillo 2008], [Callele, Neufeld, Schneider 2005], [Gomes e Wanderley 2003].

Por fim, além de analisar de que forma estes profissionais estão utilizando os processos de desenvolvimento, pretende-se identificar se estes processos possuem atividades que possibilitem a contextualização de ações educacionais na criação do jogo eletrônico educacional, elementos essenciais para a área educacional.

\section{Método de Pesquisa}

Esta é uma pesquisa qualitativa, classificada segundo seus objetivos como exploratória. Optou-se pela exploratória, pois esse tipo de classificação visa proporcionar uma visão geral acerca do fato estudado [Gil 2010], [Lakatos e Marconi 2010] e para auxiliar a coleta de informações, um questionário foi desenvolvido.

\subsection{Planejamento}

Esta etapa foi composta pelas seguintes atividades: 1. Seleção de Contexto: para essa pesquisa foram selecionados profissionais da área de desenvolvimento de jogos eletrônicos em um curso de pós-graduação específico da área; 2. Seleção dos Participantes: todos os profissionais-alunos do curso de pós-graduação em Jogos Digitais de uma universidade do sul do Brasil; e 3. Elaboração do Instrumento de Pesquisa: foi desenvolvido um questionário semiestruturado.

O questionário semiestruturado foi composto por doze questões e será apresentado no decorrer da seção Caracterização do Cenário. O questionário visou identificar o cenário estudado, na tentativa de explorar de que forma os profissionaisalunos na área de jogos eletrônicos, em especial, os educacionais estão desenvolvendo seus produtos.

Antes da aplicação, o questionário, foi avaliado por oito especialistas das áreas de: Computação (Engenharia de Software e Jogos) e Educação, e posteriormente aplicado a 27 profissionais-alunos da área de desenvolvimento de jogos eletrônicos.

\subsection{Coleta de Dados}

O método utilizado para a coleta de dados consistiu em contatar o coordenador do curso, explicando o objetivo do estudo e, em seguida, aplicar pessoalmente o questionário aos profissionais. $\mathrm{O}$ instrumento foi aplicado e recolhido no mesmo dia.

\subsection{Análise dos Resultados}

Após a aplicação do questionário, os dados foram analisados com base na pesquisa qualitativa, visando identificar se esses profissionais estão utilizando algum processo de desenvolvimento que possua alguma preocupação com a área educacional, e caso utilizem, quais são, possibilitando assim um delineamento hermenêutico do campo analisado. A próxima seção apresenta a análise dos dados. 


\section{Caracterização do Cenário}

Essa caracterização foi dividida em três grandes módulos: o primeiro módulo aborda o perfil dos participantes, das empresas e dos tipos de jogos que estes desenvolvem; o segundo módulo identifica as atividades que estes profissionais realizam durante o desenvolvimento do jogo eletrônico diverso e educacional; o terceiro módulo identifica se os profissionais que desenvolvem jogos eletrônicos educacionais possuem algum entendimento das teorias ou concepções educacionais, assim como, se há uma relação com profissionais na área de educação, que visa auxiliá-los nas questões educacionais e cognitivas.

\subsection{Módulo I - Perfil dos Participantes, Empresas e Tipos de Jogos}

Todos os participantes envolvidos na pesquisa possuem experiência profissional na área de desenvolvimento de software tradicional e de jogos eletrônicos diversos.

Em relação à experiência em desenvolvimento de jogos eletrônicos diversos, observou-se que 23 dos 27 participantes possuem experiência de até 3 anos, este tempo pode ser justificado pelo fato de ser uma área considerada nova, mostrando um crescimento considerável nos últimos anos no Brasil [Abragames 2008].

Os participantes, ao identificarem que possuem experiência em desenvolvimento de jogos eletrônicos. Vinte participantes trabalham em empresas e 7 participantes atuam como autônomos na área de desenvolvimento de jogos eletrônicos. As funções exercidas pelos profissionais nas empresas eram: gerentes de projeto (3), analistas de sistemas (9), programadores (8), designers (1) e arquitetos de software (1). O número de profissionais aqui identificados difere da quantidade apontada, pois dos (9) analistas de sistemas (5) também selecionaram a função de programadores.

Os profissionais envolvidos na pesquisa trabalham em sua maioria em empresas privadas: 19 participantes em empresas privadas e 1 participante em empresa pública.

Identificou-se durante a pesquisa que no Brasil há apenas 38 empresas cadastradas no sítio do Abragames [Abragames 2008] e a maioria dessas empresas brasileiras para jogos eletrônicos diversos e educacionais estão acopladas em empresas de desenvolvimento de softwares tradicionais.

\subsection{Módulo II - Identificação de atividades no Desenvolvimento do Jogo Eletrônico}

Esse módulo teve como objetivo identificar, mesmo para os participantes que não utilizam um processo de desenvolvimento de software, a realização de atividades independentes que contribuem para o processo no decorrer do desenvolvimento do jogo eletrônico.

\subsubsection{Método de Estimativa}

Dos 27 participantes, somente (7) utilizam algum método de estimativa. Dentre os métodos utilizados, estão: pontos por função (4 participantes); pontos por caso de uso (2 participantes); e método TXT (1 participante). O método TXT não foi identificado pela literatura como método de estimativa, desse modo, o participante deve ter confundido, pois TXT aparece posteriormente como ambiente no desenvolvimento do jogo eletrônico. 


\subsubsection{Etapas no desenvolvimento do jogo}

$\mathrm{Na}$ observação dos dados, verificou-se que 15 participantes utilizam alguma etapa no processo de desenvolvimento do jogo; já, 9 participantes não utilizam etapas; e 3 participantes não responderam esta questão. Identificou-se, por meio das respostas que a maioria dos participantes definiram algum tipo de etapa durante o desenvolvimento do jogo eletrônico.

Para uma melhor compreensão de quais etapas são trabalhadas, identificou-se que as etapas informadas pelos 15 participantes foram: game design, arte, programação, testes, documento do design, ideia, regra, Game Design Document (GDD) básico, interpretação das etapas, levantamento de funcionalidades, diagramas, fluxogramas, protótipos, utilizam SCRUM, diagramas da Unified Modeling Language (UML), além de atribuições de tarefas com brainstorming, ou seja, 56\% dos participantes utilizam algo para identificar ou documentar o desenvolvimento do jogo eletrônico.

Mesmo identificando que algumas respostas não são etapas de um processo de desenvolvimento de software, percebeu-se que os participantes não possuem conhecimento dessas atividades, visto identificá-las como etapas de desenvolvimento. Isso deve ser pelo fato de que muitos não utilizam modelos ou processos em seu contexto de trabalho, como identificado no decorrer das análises.

\subsubsection{Testes no desenvolvimento do jogo}

Tendo em vista a importância de se utilizar testes no desenvolvimento de qualquer tipo de software, estas questões tiveram como objetivo analisar se esses profissionais realizam testes durante ou na finalização do produto desenvolvido.

Verificou-se que 13 participantes realizam testes durante o processo de desenvolvimento do jogo, 11 não utilizam testes e 3 participantes não responderam esta questão. Considerou-se um número pouco expressivo pela importância da realização desses procedimentos no desenvolvimento do jogo, ou mesmo de um software ou software educacional. Dentre os testes utilizados foram identificados: playtests, caixa preta, testes de funcionalidade, unit test, testes informais, testes de usabilidade, Test Driven Development (TDD), módulo a módulo e testes realizados por colegas que fazem sugestões.

\subsubsection{Ambiente no desenvolvimento do jogo}

$\mathrm{Na}$ observação dos dados, visando identificar se esses profissionais utilizam algum ambiente para auxiliar no desenvolvimento do jogo, identificou-se que apenas 4 participantes utilizam ambiente, 19 não utilizam e 4 não responderam esta questão.

Os quatro participantes que utilizam ambientes identificaram como: Eclipse; Astah; MAC; XCODE; TXT. Os participantes destacaram que o ambiente escolhido depende do projeto a ser desenvolvido. Porém, identificou-se que esses profissionais não utilizam um ambiente específico para o desenvolvimento, mas sim plataformas existentes, e alguns confundiram ambiente como modelagem, dessa forma, observou-se que não existe, com base nas análises dessa pesquisa, um repositório ou mesmo um ambiente de informações de jogos existentes para auxiliá-los em informações de jogos que já foram desenvolvidos, possibilitando a esses profissionais, informações já desenvolvidas, evitando o retrabalho. 


\subsubsection{Processos específicos para desenvolvimento de jogos eletrônicos educacionais}

Os participantes foram questionados quanto ao conhecimento de processos específicos para desenvolvimento de jogos eletrônicos educacionais. Quatro participantes informaram ter conhecimento de processo específico e 23 desconhecem este tipo de processo.

Quando questionados quanto aos processos conhecidos, foram citados os métodos ágeis XP e SCRUM. Identificou-se que esses profissionais abordaram modelos já existentes como processos específicos para desenvolvimento de jogos eletrônicos educacionais.

\subsubsection{Utilização de processos específicos para desenvolvimento de jogos eletrônicos educacionais}

Os participantes foram questionados se utilizariam um processo específico para desenvolvimento de jogos eletrônicos educacionais, caso tivessem acesso. Dos 27 participantes, 19 responderam que utilizariam; 6 disseram que não utilizariam e 2 não responderam esta questão. Interessante observar que mesmo a maioria dos participantes não conhecendo algum processo específico para o desenvolvimento de jogos eletrônicos educacionais, como observado na subseção 4.2.5, 71\% dos entrevistados afirmaram que utilizariam um processo específico para jogos eletrônicos educacionais, direcionando, assim, para uma necessidade de ações específicas para este segmento educacional. Dentre os entrevistados que não utilizariam, acredita-se ser pelo fato de que esses profissionais desenvolvem somente jogos eletrônicos diversos e não educacionais.

\subsubsection{Fase inicial de desenvolvimento - teoria/concepção educacional}

Houve uma preocupação na pesquisa em questionar os participantes sobre a opinião em relação à importância de se considerar uma teoria ou concepções educacionais durante o desenvolvimento de jogos eletrônicos educacionais, dado a importância desse contexto no desenvolvimento deste tipo de jogo. Todos os participantes consideram importante abordar questões educacionais durante o processo de desenvolvimento do jogo eletrônico educacional, mesmo aquelas equipes que não possuem profissionais da educação trabalhando em conjunto.

Percebeu-se em estudos que, se as equipes de desenvolvimento de um software ou um jogo eletrônico educacional, objeto desta pesquisa, identificasse ou conhecesse essas ações educacionais (concepções educacionais e habilidades cognitivas), estas poderiam ser extraídas e exteriorizadas para o desenvolvimento de um jogo voltado à educação.

\subsubsection{Utilização de um processo de desenvolvimento de software}

Os participantes foram questionados sobre a utilização de algum processo de desenvolvimento de software no desenvolvimento do jogo eletrônico, e, em caso afirmativo, qual processo era utilizado. Dentre os 27 participantes, 15 relataram que utilizam algum processo de desenvolvimento de software.

Verificou-se que 15 entrevistados utilizam algum processo de desenvolvimento de software no desenvolvimento do jogo, sendo: 2 participantes utilizam o clássico, cascata ou linear-sequencial, 4 participantes o modelo iterativo, e 9 participantes metodologias ágeis. Nove entrevistados não utilizam processo de desenvolvimento. 
Com estes dados foi possível observar que são utilizados processos clássicos da engenharia de software para o desenvolvimento de jogos, e não um processo de desenvolvimento de software específico ou adaptado para esta realidade específica. Isso é um pouco preocupante para a área, pois no desenvolvimento de jogos eletrônicos, principalmente os educacionais, há uma variedade de ações que não são encontradas no desenvolvimento de software tradicionais.

\subsubsection{Mudanças no processo de desenvolvimento nos últimos três anos}

Esta questão visou identificar se houve melhoria após a inserção de algum processo de desenvolvimento nos últimos três anos. Dos 27 participantes, 10 apontaram que houve mudanças no processo de desenvolvimento de software. Os principais benefícios observados pelos profissionais foram: melhoria no tempo de desenvolvimento, melhoria na qualidade do produto final, melhoria na produtividade, custos, e redução de riscos.

As próximas análises incluem somente as respostas dos 17 profissionais que desenvolvem jogos eletrônicos educacionais, porém, em algumas análises os números podem divergir, visto que, nem todos os participantes desse módulo responderam as questões.

\subsection{Módulo III - Identificação pedagógica no desenvolvimento do jogo eletrônico educacional}

Essas análises tiveram como base o entendimento dos participantes com relação ao tipo de concepção pedagógica que é utilizada no desenvolvimento dos jogos eletrônicos educacionais, a fim de identificar se estes profissionais utilizam alguma concepção e se utilizam, qual o impacto de cada uma para o jogo eletrônico educacional desenvolvido. O conhecimento dessas concepções para o desenvolvimento de jogos eletrônicos educacionais são fundamentais para identificar a proposta de jogo que se pretende desenvolver, por isso, são apresentadas de forma sucinta o impacto de cada uma no processo de aprendizagem.

Os jogos eletrônicos educacionais formados por perguntas e respostas correspondem à concepção pedagógica Instrucionista [Valente s/d], que visa apontar a pergunta e a resposta. Por outro lado, os jogos que são formados por um conjunto de informações assemelham-se à concepção Construtivista (Piaget) [Rossler 2000]; ou Construcionista (Papert) [Papert 1985]. Essas concepções visam identificar ações que possam auxiliar a identificar e conhecer o algoritmo proposto.

As próximas questões estão relacionadas ao entendimento do tipo de jogos eletrônicos educacionais que são desenvolvidos pelos participantes.

\subsubsection{Desenvolvimento de jogos eletrônicos educacionais formados por perguntas e respostas}

Apenas 1 entrevistado desenvolve jogos eletrônicos educacionais formados por perguntas e respostas, modelo esse com uma visão instrucionista, percebe-se que, este fato pode ser considerado preocupante para o processo de desenvolvimento do jogo e para o processo de ensino e aprendizagem, visto que, esse método conforme Altoé [Altoé 2005] não atende as exigências educacionais da atualidade, pois reproduz um modelo pautado apenas na transmissão do conhecimento. 


\subsubsection{Desenvolvimento de jogos eletrônicos educacionais que utilizam um conjunto de informações}

Cinco participantes informaram que desenvolvem este tipo de jogo. Identificou-se que, dos 17 entrevistados que não utilizam o formato de pergunta e resposta, 12 entrevistados deixaram em branco esta questão porque não souberam responder.

Os questionamentos 4.3.1 e 4.3.2 visaram identificar se os profissionais compreendem quais concepções pedagógicas cada jogo corresponde, pois cada tipo de jogo possui uma especificidade para a aprendizagem. $O$ baixo índice de respostas vislumbra que a maioria dos participantes não conhece essas concepções, fato preocupante para esta área da informática na educação que desenvolve jogos eletrônicos para a aprendizagem.

\subsubsection{Definição da Teoria/Concepção Educacional no início do Processo de} Desenvolvimento de Jogos Eletrônicos Educacionais

Essa questão visou identificar se o participante define no início do processo de desenvolvimento de jogos eletrônicos educacionais, a teoria ou concepção educacional que será utilizada.

Identificou-se que: 5 participantes definem no início do processo de desenvolvimento a concepção educacional a ser utilizada e 12 participantes não definem. Quando questionados sobre qual concepção é utilizada, somente 3 responderam: 2 participantes apontaram o Construtivismo e 1 apontou o Behaviorismo.

Ao comparar esta resposta com os outros itens desse módulo, constatou-se uma disparidade entre o conjunto de respostas dos participantes, pois alguns definiram que utilizam uma concepção educacional, porém, não sabem informar qual é a concepção utilizada.

\subsubsection{Profissionais na área de educação envolvidos no processo de desenvolvimento}

Quando questionados sobre o envolvimento de profissionais da área da educação durante o processo de desenvolvimento de jogos eletrônicos educacionais, 6 participantes informaram que há profissionais da área de educação envolvidos e 9 responderam que não envolvem profissionais da área de educação no processo de desenvolvimento.

No caso do envolvimento dos profissionais da área da educação, os profissionais foram questionados sobre os benefícios com esta inclusão. Os resultados foram: 5 participantes indicaram melhoria no levantamento de requisitos; 1 participante na análise de sistema; 1 participante na implementação; 4 participantes nos testes; 3 participantes na homologação; e 1 participante, em outro, descrevendo que a melhoria consiste na: "criação do conteúdo pedagógico".

Pode-se perceber que, com a participação de um profissional da área de educação, há uma melhora significativa nas fases apontadas. Contudo, observou-se também que a ação do profissional da educação está presente em sua maioria na contextualização de etapas e no conteúdo pedagógico específico, e não na discussão das ações pedagógicas e cognitivas que o jogo eletrônico educacional proposto deve possuir. 


\section{Considerações Finais}

Essa pesquisa teve como objetivo identificar de que forma estão sendo desenvolvidos os jogos eletrônicos educacionais e se há algum processo de software específico para a área educacional.

A pesquisa aponta que os profissionais na área de desenvolvimento de jogos eletrônicos diversos e educacionais, quando adotam, utilizam modelos de processos clássicos da engenharia de software adaptados ao contexto da equipe, porém, não foi observado nenhuma discussão de questões educacionais e cognitivas nas etapas do processo de desenvolvimento de softwares desses profissionais.

Dessa forma, identificou-se a importância de ações educacionais para auxiliar os profissionais, não somente na questão de conteúdos disciplinares, como observado nas análises, mas na abstração de informações educacionais e cognitivas.

Com base nessas informações, ainda delineia-se a importância da abordagem do conhecimento das concepções pedagógicas e cognitivas durante o desenvolvimento do jogo eletrônico educacional, tendo em vista que, conhecer essas ações educacionais poderá proporcionar à equipe um direcionamento de quais procedimentos que poderão ser utilizados no decorrer do desenvolvimento de um jogo eletrônico educacional.

À vista disso, desenvolveu-se um Processo e um Ambiente computacional [Coelho Neto, Reinehr e Malucelli 2014] para auxiliar as equipes de desenvolvimento em documentar e identificar ações cognitivas para o desenvolvimento de jogos eletrônicos educacionais.

\section{Agradecimentos}

À Fundação Araucária do Estado do Paraná, pelo auxílio financeiro.

\section{Referências}

Abragames (2008) "A indústria brasileira de jogos eletrônicos: um mapeamento do crescimento do setor nos últimos 4 anos". Disponível em: http://www.abragames.org/docs/Abragames-Pesquisa2008.pdf. Acesso em: 16 jan. 2012.

Altoé, A. (2005) “Processo Tecnicista”. In: Altoé, A.; Gasparin, J. L.; Negrão, M. T. F.; Teruya, T. K. Didática: Processos de Trabalho em Sala de Aula. Eduem, pp. 65-79.

Ampatzoglou, A., e Stamelos, I. (2010) Software engineering research for computer games: A systematic review. Information and Software Technology, 52(9), Elsevier B.V., pp. 888-901.

Callele, D., Neufeld, E. e Schneider, K. (2005) "Requirements engineering and the creative process in the video game industry". In: 13th IEEE International Conference on Requirements Engineering. Washington, DC, USA, pp.240-252. 
Coelho Neto, J., Reinehr, S., Malucelli, A. (2014) "Proposal of a computerized environment to suport the development of game-based learning". In: IEEE Frontiers in Education Conference, Madrid - Spain. IEEE: IEEE, v. 1. pp. 1031-1038.

Gil, A.C. (2010) “Como elaborar Projetos de Pesquisa”. São Paulo: Editora Atlas. 5a .

Gomes, A.S. e Wanderley, E.G.(2003) "Elicitando requisitos em projetos de Software Educativo". In: Workshop Brasileiro de Informática Educativa. Campinas, SP, Brasil,: SBC. V. V. pp. 227-238.

Humphrey, W.S. (1989) "Managing the Software Process". Addison-Wesley Publishing Company, Inc., MA .

Lakatos, E.M. and Marconi, M.A. (2010) "Fundamentos de Metodologia Científica". São Paulo: Editora Atlas, $7^{\mathrm{a}}$.Ed..

Leal, G.C.L.; Stadzisz, P.C.; Almeida, C.; Perez, M.T.; Reinehr, S.; Malucelli, A. (2012) "Estudo Empírico Sobre a Avaliação da Implantação do MPS.BR em Empresas do Estado do Paraná”. In: XXXVIII CLEI. Medellin.

Papert, S. (1985) "LOGO: computadores e educação". Trad. José Armando Valente; Beatriz Bitelman; Afira Vianna Riper. São Paulo: Brasiliense.

Petrillo, F.S. (2008) "Práticas ágeis no Processo de Desenvolvimento de Jogos Eletrônicos”. 168 f. Dissertação (Mestrado em Computação) - UFRGS.

Pietruchinski, M.H; Coelho Neto, J; Malucelli, A; Reinehr, S (2011) "Os jogos educativos no contexto do SBIE: uma revisão sistemática de Literatura". In: Proceedings of XXII SBIE e XVII WIE, Aracaju-SE, Brasil, pp. 476-485.

Pinto, I.M.; Botelho, S.C.; Souza, R.C.; Goulart, T.S.; Colares, R.; Campos, R.L (2008) "Plataforma Saberlândia: Integrando Robótica e Multimídia no Desenvolvimento de Jogos Educacionais”. In: SBGAMES, Belo Horizonte, MG, Brasil, pp.177-186.

Rossler, J. H. (2000) "Construtivismo e alienação: as origens do poder de atração do ideário construtivista". Duarte, N. (org.). Sobre o construtivismo. Campinas, SP: Autores Associados, pp. 3-22.

Santos, R..A.; Góes, V.A. Almeida, L.F.(2012) "Metodologia OriGame: um processo de desenvolvimento de jogos". In: SBC Proceedings of XI SBGames - Art \& Design Track - FullPapers - Brasília - DF, pp. 125-132.

Silva, C.; Calisto, A. e Barbosa, D. (2010) "Proposta de um processo de desenvolvimento de jogos educativos". In: XXI SBIE João Pessoa, PB, pp.1-4.

Valente, J. A. (s/d) "Informática na educação: instrucionismo e Construcionismo". Disponível em: http://www.educacaopublica.rj.gov.br/biblioteca/tecnologia/0003.html. Acesso em: 23 jan. 2012. 